\title{
THE DEFORMATION OF HARMONIC MAPS GIVEN BY THE CLIFFORD TORI
}

\author{
MARIKo MUKAI
}

\section{Introduction}

The purpose of this paper is to provide some new results on deformations for harmonic maps. Let $\phi$ be a harmonic map of a compact Riemannian manifold $M$ into a Riemannian manifold $N$. A one-parameter family $\phi(t)$ of harmonic maps such that $\phi(0)=\phi$ is called a harmonic deformation of $\phi$. Then each $\phi(t)$ satisfies the harmonic map equations:

$$
\tau(\phi(t)) \equiv 0,
$$

where $\tau(\phi)$ denotes the tension field of $\phi$. By taking a derivative of the equation $(0.1)$ at $t=0$, we have the equation

$$
\left.\frac{d}{d t} \tau(\phi(t))\right|_{t=0} \equiv \mathscr{I}_{\phi}(\dot{\phi})=0, \quad \dot{\phi} \in C^{\infty}\left(\phi^{-1} T N\right) .
$$

Here $\mathscr{T}_{\phi}$ denotes the Jacobi operator of the energy functional. If a section $v \in$ $C^{\infty}\left(\phi^{-1} T N\right)$ of $\phi^{-1} T N$ satisfies the equation (0.2), then it is called an infinitesimal harmonic deformation (or a harmonic $i$-deformation) of $\phi$. We denote by $\operatorname{HID}(\phi)$ the vector space of all harmonic $i$-deformations of $\phi$. The space $\operatorname{HID}(\phi)$ just coincides with the vector space $\operatorname{Ker} \mathscr{I}_{\phi}$ of all Jacobi fields of $\phi$. If $v \in \operatorname{HID}(\phi)$ generates harmonic deformations, then $v$ is said to be integrable. Let $\operatorname{Harm}(M$, $N$ ) denote the space of all harmonic maps of $M$ into $N$. From the point of view of the deformation theory of harmonic maps, the following are fundamental problems ;

(1) to ask whether or not all harmonic $i$-deformations of $\phi$ are integrable,

(2) to make its cause clear if an harmonic $i$-deformation which is not integrable appears,

(3) to investigate the structure of a neighborhood in $\operatorname{Harm}(M, N)$ around $\phi$,

(4) to determine the connected component in $\operatorname{Harm}(M, N)$ containing $\phi$ and to examine its compactness, if it is noncompact, to construct its natural compactification.

Because of the finiteness of the dimension of $\operatorname{Ker} \mathscr{I}_{\phi}$, we know that $\operatorname{Harm}(M$, Received April 7, 1997 ; revised June 23, 1997. 
$N$ ) is locally finite dimensional (cf. [S]). For instance, Sunada [S] showed that $\operatorname{Harm}(M, N)$ is a smooth submanifold of $L_{k}^{2}(M, N)(k>m / 2, m=\operatorname{dim} M)$ with tangent space $T_{\phi} \operatorname{Harm}(M, N)=\operatorname{Ker} \mathscr{I}_{\phi}$ if $N$ is a complete locally symmetric space with non-positive sectional curvature. Certain special harmonic deformations were studied in $[\mathrm{T}]$. See also $[\mathrm{EL}],[\mathrm{K} 1]$ for other references of deformation theory for harmonic maps.

Now harmonic maps of Riemann surfaces $\Sigma$ into a compact Lie group $G$ or a compact symmetric space $G / K$ are known to have several nice properties. We have come to understand the construction and the classification of such maps, moreover, their moduli space. Hitchin $[\mathrm{H}]$ showed that the equations for a harmonic map from a 2-torus $T^{2}$ into a 3 -sphere $S^{3}$ reduce in the geometry of a spectral curve, and hence constructed the moduli space of such maps algebraic geometrically. However, it seems to be difficult to investigate the deformations for harmonic maps in general.

In this paper, to actualize the problems, we deal with harmonic maps defined by using the Clifford tori in $S^{3}$ and classify completely harmonic $i$-deformations of these maps. As we know, they are simplest and most fundamental examples of harmonic maps of $T^{2}$ into $S^{3}$. Explicitly, they are defined by $\varphi_{s}: T^{2} \rightarrow S^{3} \cong S U(2)(s \in R)$, where

$$
\varphi_{s}\left(e^{i t_{1}}, e^{i t_{2}}\right)=\left[\begin{array}{cc}
\cos s \cdot e^{i t_{1}} & \sin s \cdot e^{i t_{2}} \\
-\sin s \cdot e^{-i t_{2}} & \cos s \cdot e^{-i t_{1}}
\end{array}\right],
$$

with $t_{1}, t_{2} \in \boldsymbol{R}$. Here we endow $T^{2}$ with a conformal structure of a square. Let $\Phi_{s}:=i \circ \varphi_{s}: T^{2} \rightarrow \boldsymbol{R}^{4}$, where $i: S^{3} \hookrightarrow \boldsymbol{R}^{4}$ denotes the standard inclusion. Then we see that each $\varphi_{s}$ is harmonic, because of

$$
\Delta \Phi_{s}=-\left(\frac{\partial^{2}}{\partial t_{1}^{2}}+\frac{\partial^{2}}{\partial t_{2}^{2}}\right) \Phi_{s}=\Phi_{s}\left(=\left|d \Phi_{s}\right|^{2} \Phi_{s}\right) .
$$

Up to isometry of $S^{3}$, it is sufficient for us to deal with harmonic maps $\varphi_{s}$ with $0 \leqq s \leqq \pi / 4$. Throughout this paper, we assume that $0 \leqq s \leqq \pi / 4$ unless otherwise specified. The images of $\varphi_{s}$ with $s \neq 0$ are the Clifford tori in $S^{3}$ with constant mean curvature. In particular, the image of $\varphi_{\pi / 4}$ is the Clifford minimal torus in $S^{3}$. On the other hand, we note that when $s=0$, the image of $\varphi_{0}$ becomes a great circle in $S^{3}$. Since the group $S O(4)$ acts on $S^{3}$ as an identity component isometry group of $S^{3}$, it induces trivial harmonic deformations.

This paper is organized as follows. In Section 1, we consider the harmonic $i$-deformations in general case. The notion of infinitesimal harmonic deformations of higher order is also introduced. The notions are analogous to that of infinitesimal Einstien deformations in [K2], [K3]. Moreover, we treat the case of a harmonic map of $\Sigma$ into $G$.

In Section 2, all harmonic $i$-deformations of $\varphi_{s}$ are determined. Then an interesting jump phenomena of the dimensions of the vector spaces $\operatorname{HID}\left(\varphi_{s}\right)$ will 
occur at $s=0, \pi / 6$. In order to make the cause of this phenomena clear, we examine the behaviour of negative eigenvalues of Jacobi operator $\mathscr{I}_{\varphi_{s}}$. The index for each $\varphi_{s}$ will also be determined.

In Section 3, we classify harmonic $i$-deformations of $\varphi_{s}$. However, in cases $\varphi_{0}$ and $\varphi_{\pi / 6}$, there exist some harmonic $i$-deformations whose integrability can not be easily decided. Therefore, we shall examine whether or not they are integrable up to second order, further, up to third order. We prove the following.

Proposition 3.4, Theorems 3.6, 3.10.

(1) For $\varphi_{s}(s \neq 0, \pi / 6)$, all harmonic $i$-deformations of $\varphi_{s}$ are integrable.

(2) For $\varphi_{\pi / 6}$, there exist harmonic i-deformations of $\varphi_{\pi / 6}$ which are not integrable up to second order, and hence are not integrable.

(3) For $\varphi_{0}$, there exist harmonic $\imath$-deformations of $\varphi_{0}$ which are integrable $u p$ to second order but not up to third order, and hence are not integrable.

The results in Section 4 are the implications from those obtained in previous sections. It follows that for $s \neq 0$ a neighborhood in $\operatorname{Harm}\left(T^{2}, S^{3}\right)$ around $\varphi_{s}$, even around $\varphi_{\pi / 6}$ at which nonintegrable harmonic $i$-deformations occur, becomes a 7-dimensional smooth manifold. On the other hand, the space $\operatorname{Harm}\left(T^{2}, S^{3}\right)$ has a singular point at $\varphi_{0}$. Finally, we obtain the following.

THEOREM 4.2. The set of harmonic maps from $T^{2}$ into $S^{3}$ obtained by acting the group $S O(4)$ on the union of four families $\left\{\varphi_{s}^{\omega}\right\} \cup\left\{\tilde{\varphi}_{s}^{\omega}\right\} \cup\left\{\hat{\varphi}_{s}^{\omega}\right\} \cup\left\{\bar{\varphi}_{s}^{\omega}\right\}$ with $0 \leqq$ $s \leqq 2 \pi$ becomes a connected component in $\operatorname{Harm}\left(T^{2}, S^{3}\right)$ containing $\varphi_{s}$. Hence, the connected component is compact.

The author would like to thank Professor Y. Ohnita for his valuable suggestions and encouragement. She also would like to acknowledge their interested in this work with Professors N. Ejiri and M. Kotani and, especially, his advice on Lemma 1.1 with Professor M. Kanai.

\section{$\S 1$. The infinitesimal harmonic deformation}

We study the infinitesimal harmonic deformation (or the harmonic $i$-deformation). Let $(M, g)$ be a compact Riemannian manifold of dimension $m$ and $(N, h)$ a Riemannian manifold. First we deal with a harmonic map $\phi: M \rightarrow N$. The tension field $\tau(\phi)$ of $\phi$ is defined by $\tau(\phi)=\sum_{\imath=1}^{m}(\nabla d \phi)\left(e_{\imath}, e_{\imath}\right)$, where $\left\{e_{i}\right\}$ is an orthonormal basis on $M$. Then the harmonic map equations becomes $\tau(\phi) \equiv 0$.

Definition. A one-parameter family $\phi(t)$ of harmonic maps with $\phi(0)=\phi$ is called a harmonic deformation of $\phi$.

DEFinition. A harmonic deformation $\phi(t)$ of $\phi$ is said to be trivial if there exist isometries $\sigma_{M}(t), \sigma_{N}(t)$ of $M, N$ with $\sigma_{M}(0)=\mathrm{id}_{M}, \sigma_{N}(0)=\mathrm{id}_{N}$ such that $\phi(t)$ $=\sigma_{N}(t)^{-1} \circ \phi \circ \sigma_{M}(t)$. 
Assume that $\phi(t)$ is a deformation of $\phi$. If we express the Taylor expansion of $\tau(\phi(t))$ as

$$
\tau(\phi(t))=c_{0}(\phi)+t c_{1}(\dot{\phi})+\frac{t^{2}}{2 !} c_{2}(\dot{\phi}, \ddot{\phi})+\frac{t^{3}}{3 !} c_{3}(\dot{\phi}, \ddot{\phi}, \dddot{\phi})+\cdots,
$$

where

$$
\dot{\phi}=\left.\frac{\partial \phi(t)}{\partial t}\right|_{t=0}, \quad \ddot{\phi}=\left.\nabla_{\partial / \partial t}^{N} \frac{\partial \phi(t)}{\partial t}\right|_{t=0}, \quad \dddot{\phi}=\left.\nabla_{\partial / \partial t}^{N} \nabla_{\partial / \partial}^{N} \frac{\partial \phi(t)}{\partial t}\right|_{t=0} \in C^{\infty}\left(\phi^{-1} T N\right),
$$

then the equation $\tau(\phi(t))=0$ implies the following system of the equations:

$$
c_{0}(\phi)(=\tau(\phi)) \equiv 0, \quad c_{1}(\dot{\phi})=0, \quad c_{2}(\dot{\phi}, \ddot{\phi})=0, \quad c_{3}(\dot{\phi}, \ddot{\phi}, \dddot{\phi})=0, \ldots
$$

By the direct computation, we have the following.

LEMMA 1.1. Under the above situation, for a harmonic map $\phi: M \rightarrow N$, we have

Here

$$
\begin{gathered}
c_{1}(\dot{\phi})=-\mathscr{I}_{\phi}(\dot{\phi}), \\
c_{2}(\dot{\phi}, \ddot{\phi})=-I_{\phi}(\ddot{\phi})+4 \sum_{i=1}^{m} R^{N}\left(\dot{\phi}, d \phi\left(e_{\imath}\right)\right)\left(\nabla_{e_{i}}^{\phi} \dot{\phi}\right) \\
+\left[\text { terms of } \nabla R^{N}\right] \\
c_{3}(\dot{\phi}, \ddot{\phi}, \dddot{\phi})=-I_{\phi}(\dddot{\phi})+6 \sum_{\imath=1}^{m} R^{N}\left(\ddot{\phi}, d \phi\left(e_{\imath}\right)\right)\left(\nabla_{e_{i}}^{\phi} \dot{\phi}\right) \\
+6 \sum_{\imath=1}^{m} R^{N}\left(\dot{\phi}, d \phi\left(e_{\imath}\right)\right)\left(\nabla_{e_{i}}^{\phi} \ddot{\phi}\right)+4 \sum_{\imath=1}^{m} R^{N}\left(\dot{\phi}, \nabla_{e_{i}}^{\phi} \dot{\phi}\right)\left(\nabla_{e_{i}}^{\phi} \dot{\phi}\right) \\
+4 \sum_{i=1}^{m} R^{N}\left(\dot{\phi}, d \phi\left(e_{\imath}\right)\right)\left(R^{N}\left(\dot{\phi}, d \phi\left(e_{\imath}\right)\right) \dot{\phi}\right) \\
+\left[\operatorname{terms~of~} \nabla R^{N}, \nabla \nabla R^{N}\right] .
\end{gathered}
$$

$$
\mathscr{I}_{\phi} v=\left(\nabla^{\phi}\right)^{*}\left(\nabla^{\phi}\right) v-\sum_{\imath=1}^{m} R^{N}\left(v, d \phi\left(e_{\imath}\right)\right) d \phi\left(e_{\imath}\right), \quad v \in C^{\infty}\left(\phi^{-1} T N\right)
$$

is the Jacobi operator of $\phi$, where $\left(\nabla^{\phi}\right)^{*}\left(\nabla^{\phi}\right)$ denotes the rough Laplacian with respect to the connection $\nabla^{\phi}$.

Now let us review here the Jacobi operator $\mathscr{I}_{\phi}$ in the above lemma from the point of view of the variational problem. It is a self-adjoint linear elliptic differential operator, which appears in the second variational formula of the energy for a harmonic map $\phi$. We have $C^{\infty}\left(\phi^{-1} T N\right)=\oplus_{\lambda} V_{\lambda}$, where $V_{\lambda}$ is the eigenspace of $\mathscr{I}_{\phi}$ with eigenvalue $\lambda$. Then the nullity and index of $\phi$ are defined by 


$$
\begin{gathered}
\operatorname{nullity}(\phi)=\operatorname{dim} V_{0}=\operatorname{dim} \operatorname{Ker} \mathscr{I}_{\phi}, \\
\operatorname{index}(\phi)=\sum_{\lambda<0} \operatorname{dim} V_{\lambda},
\end{gathered}
$$

respectively. We note that each number is finite. A harmonic map $\phi$ is said to be stable if index $(\phi)=0$.

Then we return to the discussion of harmonic $i$-deformations of $\phi$.

DEFINITION. Let $\phi: M \rightarrow N$ be a harmonic map. We call that

(1) a section $v$ of $\phi^{-1} T N$ is a harmonic $i$-deformation of $\phi$ if $c_{1}(v)=0$;

(2) a pair $(v, w)$ of sections of $\phi^{-1} T N$ is a harmonic i-deformation of second order of $\phi$ if $v$ is a harmonic $i$-deformation of $\phi$ and $c_{2}(v, w)=0$;

(3) a triple $(v, w, z)$ of sections of $\phi^{-1} T N$ is a harmonic $i$-deformation of third order of $\phi$ if $(v, w)$ is a harmonic $i$-deformation of second order of $\phi$ and $c_{3}(v, w, z)=0$.

DEFINITION. A harmonic $i$-deformation $v$ of $\phi$ is said to be trivial if $v$ is of the form of a linear combination of $d \phi(X)$ and $Y \circ \phi$. Here $X$ is a Killing vector field on $M$ if $\operatorname{dim} M \neq 2$, or a conformal vector field if $\operatorname{dim} M=2$, and $Y$ is a Killing vector filed on $N$.

We denote by $\operatorname{HID}(\phi)$ the vector space of all harmonic $i$-deformations of $\phi$. Then it follows that

$$
\operatorname{dim} \operatorname{HID}(\phi)=\operatorname{nullity}(\phi) .
$$

DEFINITION. Let $v$ be a harmonic $i$-deformation of a harmonic map $\phi$ : $M \rightarrow N$.

(1) If there exists $w \in C^{\infty}\left(\phi^{-1} T N\right)$ such that $(v, w)$ becomes a harmonic $i$ deformation of second order of $\phi$, then $v$ is said to be integrable up to second order.

(2) If there exist $w, z \in C^{\infty}\left(\phi^{-1} T N\right)$ such that $(v, w, z)$ becomes a harmonic $i$-deformation of third order of $\phi$, then $v$ is said to be integrable up to third order.

Definition. Let $\phi: M \rightarrow N$ be a harmonic map. A harmonic $i$-deformation $v$ of $\phi$ is said to be integrable if there exists a harmonic deformation $\phi(t)$ of $\phi$ such that

$$
v=\left.\frac{\partial \phi(t)}{\partial t}\right|_{t=0} .
$$

Obviously, the following holds.

LEMMA 1.2. If a harmonic i-deformation $v$ is not integrable up to second or third order, then $v$ is not integrable.

Next we deal with a harmonic map $\phi$ from a Riemann surface $\Sigma$ into a 
Lie group $G$ with Lie algebra g. Indeed, we are more intereseted in harmonic $i$-deformations of such maps. In this case, we can describe harmonic $i$-deformations Lie algebraically. The harmonic map equation becomes

$$
d * \theta=0 \text {. }
$$

Here $\theta:=\phi^{-1} d \phi$ denotes the pull-back of Maurer-Cartan form of $G$, which is a 1 -form with values in $\phi^{-1} T G=\underline{g}$. Let $\phi(t)$ be a harmonic deformation of $\phi$. Put $\theta_{t}=\phi(t)^{-1} d \phi(t)$. Then it is convenient to use the following expansion instead of $(1.1)$ :

$$
d * \theta_{t}=a_{0}+t a_{1}(\xi)+\frac{t^{2}}{2 !} a_{2}(\xi, \dot{\xi})+\frac{t^{3}}{3 !} a_{3}(\xi, \dot{\xi}, \ddot{\xi})+\cdots,
$$

in terms of $\xi, \dot{\xi}=\left.\left(d \xi_{t} / d t\right)\right|_{t=0}$ and $\ddot{\xi}=\left.\left(d^{2} \xi_{t} / d t^{2}\right)\right|_{t=0}$, where

$$
\xi_{t}=\phi(t)^{-1} \frac{\partial \phi(t)}{\partial t} \in C^{\infty}(\underline{g})
$$

with $\xi_{0}=\xi$. Note that the order of derivative of $\xi$ in (1.9) is one order less than that of $\phi$ in (1.1). We see that $a_{0}=d * \theta \equiv 0$. The Jacobi operator in the following lemma is of the form

$$
\mathscr{I}_{\phi}(\zeta)=-* d * d_{\theta} \zeta, \quad \text { for } \zeta \in C^{\infty}(\underline{\mathfrak{g}}),
$$

with $d_{\theta}=d+\operatorname{ad} \theta$.

LEMMA 1.3. Under the above situation, we have

$$
\begin{gathered}
a_{1}(\xi)=-* \mathscr{I}_{\phi}(\xi), \\
a_{2}(\xi, \dot{\xi})=-* \mathscr{I}_{\phi}(\dot{\xi})-P(\xi), \\
a_{3}(\xi, \dot{\xi}, \ddot{\xi})=-* \mathscr{I}_{\phi}(\ddot{\xi})-Q(\xi, \dot{\xi}),
\end{gathered}
$$

putting

$$
P(\xi):=[[* \theta, \xi] \wedge d \xi],
$$

(1.15) $Q(\xi, \dot{\xi}):=[* d \xi \wedge d \dot{\xi}]+2[[* \theta, \xi] \wedge d \dot{\xi}]+[[* \theta, \dot{\xi}] \wedge d \xi]+\left[\left[* d_{\theta} \xi, \xi\right] \wedge d \xi\right]$.

Proof. Since $\left(d \theta_{t} / d t\right)=d_{\theta_{t}} \xi_{t}$, we get

$$
\frac{d}{d t}\left(d * \theta_{t}\right)=d * d_{\theta_{t}} \xi_{t},
$$

and hence, at $t=0, a_{1}(\xi)=\left.(d / d t)\left(d * \theta_{t}\right)\right|_{t=0}=-* \mathscr{I}_{\phi}(\xi)$. Using

$$
\frac{d}{d t}\left(d_{\theta_{t}} \xi_{t}\right)=d_{\theta_{t}} \frac{d \xi_{t}}{d t}+\left[d_{\theta_{t}} \xi_{t}, \xi_{t}\right] \text {, }
$$

we have 


$$
\begin{aligned}
& \frac{d}{d t}\left(d * d_{\theta_{t}} \xi_{t}\right)=d * d_{\theta_{t}} \frac{d \xi_{t}}{d t}+\left[d * d_{\theta_{t}} \xi_{t}, \xi_{t}\right]-\left[* d_{\theta_{t}} \xi_{t} \wedge d \xi_{t}\right\rfloor \\
& =d * d_{\theta_{t}} \frac{d \xi_{t}}{d t}-\left[* d_{\theta_{t}} \xi_{t} \wedge d \xi_{t}\right] \quad \text { because } d * d_{\theta_{t}} \xi_{t}=0 \\
& =d * d_{\theta_{t}} \frac{d \xi_{t}}{d t}-\left[\left[* \theta_{t}, \xi_{t}\right] \wedge d \xi_{t}\right] \quad \text { because }\left[* d \xi_{t} \wedge d \xi_{t}\right]=0
\end{aligned}
$$

By putting $t=0$, we have (1.12). Moreover, we compute

$$
\begin{aligned}
& \frac{d}{d t}\left(d * d_{\theta_{t}} \frac{d \xi_{t}}{d t}-\left[\left[* \theta_{t}, \xi_{t}\right] \wedge d \xi_{t}\right]\right) \\
& =d * d_{\theta_{t}} \frac{d^{2} \xi_{t}}{d t^{2}}-\left[* d_{\theta_{t}} \xi_{t} \wedge d\left(\frac{d \xi_{t}}{d t}\right)\right] \\
& \quad-\left[\left[* d_{\theta_{t}} \xi_{t}, \xi_{t}\right] \wedge d \xi_{t}\right]-\left[\left[* \theta_{t}, \frac{d \xi_{t}}{d t}\right] \wedge d \xi_{t}\right]-\left[\left[* \theta_{t}, \xi_{t}\right] \wedge d\left(\frac{d \xi_{t}}{d t}\right)\right] .
\end{aligned}
$$

Hence at $t=0$,

$$
\begin{aligned}
& \left.\frac{d}{d t}\left(d * d_{\theta_{t}} \frac{d \xi_{t}}{d t}-\left[\left[* \theta_{t}, \xi_{t}\right] \wedge d \xi_{t}\right]\right)\right|_{t=0} \\
= & d * d_{\theta} \ddot{\xi}-\left[* d_{\theta} \xi \wedge d \dot{\xi}\right] \\
& -\left[\left[* d_{\theta} \xi, \xi\right] \wedge d \xi\right]-[[* \theta, \dot{\xi}] \wedge d \xi]-[[* \theta, \xi] \wedge d \dot{\xi}] \\
= & -* I_{\phi}(\ddot{\xi}) \\
& -\left([* d \xi \wedge d \dot{\xi}]+2[[* \theta, \xi] \wedge d \dot{\xi}]+[[* \theta, \dot{\xi}] \wedge d \xi]+\left[\left[* d_{\theta} \xi, \xi\right] \wedge d \xi\right]\right) .
\end{aligned}
$$

DEFINITION. For a harmonic map $\phi: \Sigma \rightarrow G$, we call that

(1) a section $\xi$ of $g$ is a harmonic $i$-deformation of $\phi$ if $a_{1}(\xi)=0$;

(2) a pair $(\xi, \eta)$ of sections of $\mathfrak{g}$ is a harmonic $i$-deformation of second order of $\phi$ if $\xi$ is a harmonic $i$-deformation of $\phi$ and $a_{2}(\xi, \eta)=0$;

(3) a triple $(\xi, \eta, \gamma)$ of sections of $g$ is a harmonic $i$-deformation of third order of $\phi$ if $(\xi, \eta)$ is a harmonic $i$-deformation of second order of $\phi$ and $a_{3}(\xi$, $\eta, \gamma)=0$ $\Sigma \rightarrow G$.

DEFINITION. Let $\xi$ be a harmonic $i$-deformation of a harmonic map $\phi$ :

(1) If there exists $\eta \in C^{\infty}(\underline{g})$ such that $(\xi, \eta)$ becomes a harmonic $i$-deformation of second order of $\phi$, then $\xi$ is said to be integrable up to second order.

(2) If there exist $\eta, \gamma \in C^{\infty}(\underline{g})$ such that $(\xi, \eta, \gamma)$ becomes a harmonic $i$-deformation of third order of $\phi$, then $\xi$ is said to be integrable up to third order. 
Definition. Let $\phi: \Sigma \rightarrow G$ be a harmonic map. A harmonic $i$-deformation $\xi$ of $\phi$ is said to be integrable if there exists a harmonic deformation $\phi(t)$ of $\phi$ such that

$$
\xi=\left.\phi^{-1} \frac{\partial \phi(t)}{\partial t}\right|_{t=0}
$$

By Lemma 1.3, the following is obvious.

LEMMA 1.4. Let $\phi: \Sigma \rightarrow G$ be a harmonic map and $\xi$ a harmonic $i$-deformation of $\phi$. Then

(1) $\xi$ is integrable up to second order if and only if $* P(\xi)$ is orthogonal to $\operatorname{Ker} \mathscr{T}_{\phi}$.

(2) $\xi$ is integrable up to third order if and only if $* Q(\xi, \eta)$ is orthogonal to $\operatorname{Ker} \mathscr{T}_{\phi}$ for some $\eta$ such that $a_{2}(\xi, \eta)=0$.

Here we use the $L^{2}$-inner product on $C^{\infty}(\underline{g})$.

\section{§2. The infinitesimal harmonic deformations of $\varphi_{s}$}

Let us focus on harmonic maps $\varphi_{s}: T^{2} \rightarrow S U(2)$ defined by using the Clifford tori in (0.3). We begin by determining completely the harmonic $i$-deformations of $\varphi_{s}$. In this section, it is sufficient to take $0 \leqq s \leqq \pi / 4$. From (1.10) and (1.11), the equation $a_{1}(\xi)=0$ of the harmonic $i$-deformations becomes

$$
\frac{\partial^{2} \xi}{\partial t_{1}^{2}}+\frac{\partial^{2} \xi}{\partial t_{2}^{2}}+\left[\left(\theta_{s}\right)_{1}, \frac{\partial \xi}{\partial t_{1}}\right]+\left[\left(\theta_{s}\right)_{2}, \frac{\partial \xi}{\partial t_{2}}\right]=0, \quad \text { for } \quad \xi \in C^{\infty}(\underline{g})
$$

Here the pull-back $\theta_{s}=\varphi_{s}{ }^{-1} d \varphi_{s}$ of Maurer-Cartan form of $G=S U(2)$ by $\varphi_{s}$ is spliting as $\theta_{s}=\left(\theta_{s}\right)_{1} d t_{1}+\left(\theta_{s}\right)_{2} d t_{2}$, where

$$
\begin{aligned}
& \left(\theta_{s}\right)_{1}=i\left[\begin{array}{cc}
\cos ^{2} s & \cos s \sin s \cdot e^{-i t_{1}} e^{i t_{2}} \\
\cos s \sin s \cdot e^{i t_{1}} e^{-i t_{2}} & -\cos ^{2} s
\end{array}\right], \\
& \left(\theta_{s}\right)_{2}=i\left[\begin{array}{cc}
-\sin ^{2} s & \cos s \sin s \cdot e^{-i t_{1}} e^{-i t_{2}} \\
\cos s \sin s \cdot e^{i t_{1}} e^{-i t_{2}} & \sin ^{2} s
\end{array}\right] .
\end{aligned}
$$

Proposition 2.1. The vector space $\operatorname{HID}\left(\varphi_{s}\right)$ of harmonic 2-deformations of $\varphi_{s}$ becomes

$$
\operatorname{HID}\left(\varphi_{s}\right)= \begin{cases}\sum_{i=1}^{7} \boldsymbol{R} \xi_{\imath}, & \text { if } s \neq 0, \pi / 6, \\ \sum_{\imath=1}^{9} \boldsymbol{R} \xi_{\imath}, & \text { if } s=\pi / 6, \\ \sum_{\imath=1}^{7} \boldsymbol{R} \xi_{i}+\sum_{\imath=8}^{9} \boldsymbol{R} \tilde{\xi}_{\imath}, & \text { if } s=0 .\end{cases}
$$

Here $\xi_{\imath}(i=1, \ldots, 9)$ and $\tilde{\xi}_{\imath}(i=8,9)$ are defined as 


$$
\begin{aligned}
& \xi_{1}=\left[\begin{array}{cc}
i & 0 \\
0 & -i
\end{array}\right], \quad \xi_{2}=\left[\begin{array}{ll}
0 & i \\
i & 0
\end{array}\right], \quad \xi_{3}=\left[\begin{array}{cc}
0 & 1 \\
-1 & 0
\end{array}\right], \\
& \xi_{4}=i\left[\begin{array}{cc}
-\tan s \cdot\left(e^{-i t_{1}} e^{-i t_{2}}+e^{i t_{1}} e^{i t_{2}}\right) & e^{-2 i t_{1}}-\tan ^{2} s \cdot e^{2 i t_{2}} \\
e^{2 i t_{1}}-\tan ^{2} s \cdot e^{-2 i t_{2}} & \tan s \cdot\left(e^{-i t_{1}} e^{-i t_{2}}+e^{i t_{1}} e^{i t_{2}}\right)
\end{array}\right] \text {, } \\
& \xi_{5}=\left[\begin{array}{cc}
-\tan s \cdot\left(e^{-i t_{1}} e^{-i t_{2}}-e^{i t_{1}} e^{i t_{2}}\right) & e^{-2 i t_{1}}+\tan ^{2} s \cdot e^{2 i t_{2}} \\
-e^{2 i t_{1}}-\tan ^{2} s \cdot e^{-2 i t_{2}} & \tan s \cdot\left(e^{-i t_{1}} e^{-i t_{2}}-e^{i t_{1}} e^{i t_{2}}\right)
\end{array}\right], \\
& \xi_{6}=i\left[\begin{array}{cc}
0 & e^{-i t_{1}} e^{i t_{2}} \\
e^{i t_{1}} e^{-i t_{2}} & 0
\end{array}\right], \quad \xi_{7}=\left[\begin{array}{cc}
0 & e^{-i t_{1}} e^{i t_{2}} \\
-e^{i t_{1}} e^{-i t_{2}} & 0
\end{array}\right] \text {, } \\
& \xi_{8}=i\left[\begin{array}{cc}
\tan s \cdot\left(e^{-i t_{1}}+e^{i t_{1}}\right) & e^{i t_{2}}-\tan ^{2} s \cdot e^{-2 i t_{1}} e^{i t_{2}} \\
e^{-i t_{2}}-\tan ^{2} s \cdot e^{2 i t_{1}} e^{-i t_{2}} & -\tan s \cdot\left(e^{-i t_{1}}+e^{i t_{1}}\right)
\end{array}\right] \text {, } \\
& \xi_{9}=\left[\begin{array}{cc}
\tan s \cdot\left(e^{-i t_{1}}+e^{i t_{1}}\right) & e^{i t_{2}}+\tan ^{2} s \cdot e^{-2 i t_{1}} e^{i t_{2}} \\
-e^{-i t_{2}}-\operatorname{ant}^{2} s \cdot e^{2 i t_{1}} e^{-i t_{2}} & -\tan s \cdot\left(e^{-i t_{1}}+e^{i t_{1}}\right)
\end{array}\right], \\
& \tilde{\xi}_{8}=i\left[\begin{array}{cc}
0 & e^{-i t_{1}} e^{-i t_{2}} \\
e^{i t_{1}} e^{i t_{2}} & 0
\end{array}\right], \quad \tilde{\xi}_{9}=\left[\begin{array}{cc}
0 & e^{-i t_{1}} e^{-i t_{2}} \\
-e^{i t_{1}} e^{i t_{2}} & 0
\end{array}\right] \text {. }
\end{aligned}
$$

Proof. By regarding $\xi=\left[\begin{array}{cc}a & b \\ c & -a\end{array}\right]$ as an $\mathfrak{g} \mathfrak{u}(2)^{c}(=\mathfrak{g l}(2, C))$-valued function on $T^{2}$, we express a Fourier expansion of $\xi$ as $\xi=\sum_{k, l \in Z} \xi_{k l} e^{i k t_{1}} e^{i l t_{2}}, \xi_{k l}=\left[\begin{array}{ll}a_{k l} & b_{k l} \\ c_{k l} & -a_{k l}\end{array}\right]$. Then the equation (2.1) is equivalent to the system of linear algebraic equations with unknowns $a_{k l}, b_{k l}, c_{k l}$ and parameter $s$ :

$$
\left\{\begin{array}{l}
\left(k^{2}+l^{2}\right) a_{k l}-(k+l) \cos s \sin s \cdot\left(b_{k-1 l+1}-c_{k+1 l-1}\right)=0, \\
2(k+l) \cos s \sin s \cdot a_{k+1 l-1}-\left\{\left(k^{2}+l^{2}\right)+2\left(k \cos ^{2} s-l \sin ^{2} s\right)\right\} b_{k l}=0, \\
2(k+l) \cos s \sin s \cdot a_{k-1 l+1}+\left\{\left(k^{2}+l^{2}\right)-2\left(k \cos ^{2} s-l \sin ^{2} s\right)\right\} c_{k l}=0 .
\end{array}\right.
$$

We need a long calculation to solve the equations (2.5). Then setting

$$
\begin{gathered}
a_{0}:=a_{00}, \quad b_{0}:=b_{00}, \quad c_{0}:=c_{00}, \quad b_{1}:=b_{-11}, \quad c_{1}:=c_{1-1}, \\
b_{2}:=b_{-20}, \quad c_{2}:=c_{20}, \quad b_{3}:=b_{-1-1}, \quad c_{3}:=c_{11}, \\
b_{4}:=b_{02} \text { and } c_{4}:=c_{0-2}
\end{gathered}
$$

for simplicity, we obtain that each $a, b, c$ is of the following form;

if $s \neq 0, \pi / 6$, 


$$
\left\{\begin{array}{l}
a=a_{0}-b_{2} \tan s \cdot e^{-i t_{1}} e^{-i t_{2}}-c_{2} \tan s \cdot e^{i t_{1}} e^{i t_{2}} \\
b=b_{0}+b_{2} e^{-2 i t_{1}}+b_{1} e^{-i t_{1}} e^{i t_{2}}-c_{2} \tan ^{2} s \cdot e^{2 i t_{2}} \\
c=c_{0}+c_{2} e^{2 i t_{1}}+c_{1} e^{i t_{1}} e^{-i t_{2}}-b_{2} \tan ^{2} s \cdot e^{-2 i t_{2}}
\end{array}\right.
$$

if $s=\pi / 6$,

$$
\left\{\begin{array}{l}
a=a_{0}-b_{2} \tan s \cdot e^{-i t_{1}} e^{-i t_{2}}-c_{2} \tan s \cdot e^{i t_{1}} e^{i t_{2}}+c_{4} \tan s \cdot e^{-i t_{1}}+b_{4} \tan s \cdot e^{i t_{1}} \\
b=b_{0}+b_{2} e^{-2 i t_{1}}+b_{1} e^{-i t_{1}} e^{i t_{2}}-c_{2} \tan ^{2} s \cdot e^{2 i t_{2}}-c_{4} \tan ^{2} s \cdot e^{-2 i t_{1}} e^{i t_{2}}+b_{4} e^{2 t_{2}} \\
c=c_{0}+c_{2} e^{2 i t_{1}}+c_{1} e^{i t_{1}} e^{-i t_{2}}-b_{2} \tan ^{2} s \cdot e^{-2 i t_{2}}-b_{4} \tan ^{2} s \cdot e^{2 i t_{1}} e^{-i t_{2}}+c_{4} e^{-i t_{2}}
\end{array}\right.
$$

if $s=0$,

$$
\left\{\begin{array}{l}
a=a_{0} \\
b=b_{0}+b_{2} e^{-22 t_{1}}+b_{1} e^{-i t_{1}} e^{i t_{2}}+b_{3} e^{-i t_{1}} e^{-i t_{2}} \\
c=c_{0}+c_{2} e^{2 i t_{1}}+c_{1} e^{i t_{1}} e^{-i t_{2}}+c_{3} e^{i t_{1}} e^{2 t_{2}}
\end{array}\right.
$$

Using the conditions $a \in \sqrt{-1} R$ and $b=-\bar{c}$ of $\xi \in \xi \mathfrak{z}(2)$, we can show that $\xi \in$ $\operatorname{HID}\left(\varphi_{s}\right)$ can be written as a linear combination of $\xi_{\imath}$ or $\tilde{\xi}_{2}$. Hence we complete the proof of Proposition 2.1.

Consequently, we have determined the dimension of each vector space $\operatorname{HID}\left(\varphi_{s}\right)$, namely, the nullity of $\varphi_{s}$ as a harmonic map from (1.8). Then we encounter an interesting jump phenomena of the numbers.

COROLlary 2.2. We have

$$
\operatorname{dim} \operatorname{HID}\left(\varphi_{s}\right)= \begin{cases}9, & \text { if } s=0, \pi / 6, \\ 7, & \text { otherwise, }\end{cases}
$$

(see Figuer 2.1(i)).

To make the cause of this jump phenomena at $s=0$ and $\pi / 6$ clear, we next examine the behaviour of the negative eigenvalues of $\mathscr{I}_{\varphi_{\boldsymbol{\delta}}}$.

Proposition 2.3. Take $s$ with $0 \leqq s \leqq \pi / 2$. Each Jacobi operator $\mathscr{T}_{\varphi_{\boldsymbol{s}}}$ has negative eigenvalues

$$
\begin{cases}\lambda_{1}:=1-\sqrt{2(1+\cos 2 s)}, & \text { if } 0 \leqq s \leqq \pi / 3, \\ \lambda_{2}:=1-\sqrt{2(1-\cos 2 s),} & \text { if } \pi / 6 \leqq s \leqq \pi / 2,\end{cases}
$$

with multiplicity 2(see Figuer 2.1(ii)). Moreover, the eigenspace corresponding to $\lambda_{2}$ is spanned by $\xi_{8}, \xi_{9}$.

Proof. Suppose $\lambda<0$. Similar to the proof of Proposition 2.1, we find that 
the equations $\mathscr{T}_{\varphi_{s}}(\xi)=\lambda \xi$ are equivalent to the system of linear algebraic equations with unknowns $a_{k l}, b_{k l}, c_{k l}$, and parameters $s$ and $\lambda$ :

$$
\left\{\begin{array}{l}
\left(k^{2}+l^{2}-\lambda\right) a_{k l}-(k+l) \cos s \sin s \cdot\left(b_{k-1 l+1}-c_{k+1 l-1}\right)=0, \\
2(k+l) \cos s \sin s \cdot a_{k+1 l-1}-\left\{\left(k^{2}+l^{2}\right)+2\left(k \cos ^{2} s-l \sin ^{2} s\right)-\lambda\right\} b_{k l}=0, \\
2(k+l) \cos s \sin s \cdot a_{k-1 l+1}+\left\{\left(k^{2}+l^{2}\right)-2\left(k \cos ^{2} s-l \sin ^{2} s\right)-\lambda\right\} c_{k l}=0 .
\end{array}\right.
$$

By a harder calculation than that for (2.5), we find that $a_{k l}=0$ for except $(k, l)$ satisfying either (2.10) or (2.11) of the following:

$$
\begin{gathered}
l=0 \text { and } \lambda^{2}-2 k^{2} \lambda+k^{4}-2 k^{2}(1-\cos 2 s)=0 \\
l^{2}=1 \text { and } \lambda^{2}-2\left(k^{2}+1\right) \lambda+k^{4}+2 k^{2} \cos 2 s-1-2 \cos 2 s=0,
\end{gathered}
$$

which are equivalent to

$$
\begin{gathered}
l=0 \text { and } \lambda=k^{2}-\sqrt{2 k^{2}(1-\cos 2 s)} \\
l^{2}=1 \text { and } \lambda=\left(k^{2}+1\right)-\sqrt{2\left(k^{2}-k^{2} \cos 2 s+1+\cos 2 s\right),}
\end{gathered}
$$$$
\text { respectively. Then it follows from } \lambda<0 \text { and } k \in \boldsymbol{Z} \text { that }
$$

$$
\begin{aligned}
& (k, l, \lambda)=( \pm 1,0,1-\sqrt{2(1-\cos 2 s)}) \\
& (k, l, \lambda)=(0, \pm 1,1-\sqrt{2(1+\cos 2 s)}),
\end{aligned}
$$

respectively. In particular, put $\lambda_{2}:=1-\sqrt{2(1-\cos 2 s)}$. Using (2.9) again, we obatin that each $a, b, c$ in $\xi=\left[\begin{array}{cc}a & b \\ c & -a\end{array}\right]$ satisfying $\mathscr{I}_{\varphi_{s}}(\xi)=\lambda_{2}(\xi)$ is of the form:

$$
\left\{\begin{array}{l}
a=c_{4} \tan s \cdot e^{-i t_{1}}+b_{4} \tan s \cdot e^{i t_{1}} \\
b=-c_{4} \tan ^{2} s \cdot e^{-2 i t_{1}} e^{i t_{2}}+b_{4} e^{i t_{2}} \\
c=-b_{4} \tan ^{2} s \cdot e^{2 i t_{1}} e^{-i t_{2}}+c_{4} e^{-i t_{2}}
\end{array}\right.
$$

By virtue of Proposition 2.3, it follows that the subspace of $\operatorname{HID}\left(\varphi_{\pi / 6}\right)$ which cause the jump phenomena of dimension 2 just coincides with the eigenspace corresponding to $\lambda_{2}$. The index of each $\varphi_{s}$ can be also determined.

COROLLARY 2.4. Take s with $0 \leqq s \leqq \pi / 2$. We have

$$
\operatorname{index}\left(\varphi_{s}\right)= \begin{cases}2, & \text { if } 0 \leqq s \leqq \pi / 6, \pi / 3 \leqq s \leqq \pi / 2, \\ 4, & \text { if } \pi / 6<s<\pi / 3,\end{cases}
$$

(see Figure 2.1(iii)).

Remark. Proposition 2.3 implies that the harmonic maps from $T^{2}$ into 3dimensional real projective space $R P^{3}$ obtained by compositions of $\left\{\varphi_{s}\right\}$ and the 
natural projection $S^{3} \rightarrow \boldsymbol{R} P^{3}$ are stable in the sense of index=0. Very recently Ejiri proves in $[\mathrm{Ej}]$ that the maps become non-zero homotopically energy minimizing. Thus our result supports Ejiri's one.

(i) nullity

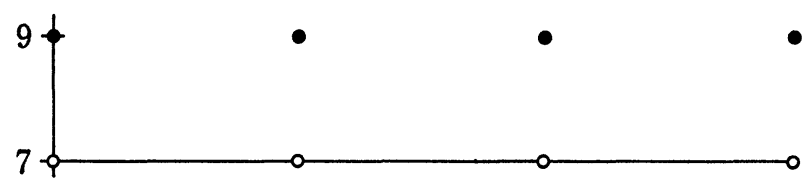

(ii) $\lambda$


Figure 2.1.

\section{§3. Classification of infinitesimal harmonic deformations}

In this section, assume that $0 \leqq s \leqq \pi / 4$. It is easy to check the following.

LEMMA 3.1. Let $f_{(a, b)}: T^{2} \ni\left(e^{2 t_{1}}, e^{i t_{2}}\right) \mapsto\left(e^{\imath\left(t_{1}+a\right)}, e^{i\left(t_{2}+b\right)}\right) \in T^{2}$ be a conformal transformation of $T^{2}$. Let $R$ and $L$ denote right and left translations of $S U(2)$, respectively. Then

$$
\varphi_{s} \circ f_{(a, b)}\left(e^{i t_{1}}, e^{i t_{2}}\right)=\left(R_{A} \circ L_{B^{\circ}} \varphi_{s}\right)\left(e^{i t_{1}}, e^{i t_{2}}\right),
$$

where $A=\left[\begin{array}{cc}e^{\imath \alpha} & 0 \\ 0 & e^{-\imath \alpha}\end{array}\right], B=\left[\begin{array}{cc}e^{\imath \beta} & 0 \\ 0 & e^{-\imath \beta}\end{array}\right] \in S U(2)$, putting $\alpha=(a+b) / 2, \beta=(a-b) / 2$.

LEMMA 3.2. Trivial harmonic i-deformations of $\varphi_{s}$ span

$$
\begin{cases}W_{0}:=\sum_{\imath=1}^{6} \boldsymbol{R} \xi_{\imath}, & \text { if } s \neq 0, \\ V_{0}:=\sum_{\imath=1}^{5} \boldsymbol{R} \xi_{\imath}, & \text { if } s=0 .\end{cases}
$$

Proof. By Lemma 3.1, a trivial harmonic $i$-deformation of $\varphi_{s}$ is of the form $Y \circ{ }^{\circ} \varphi_{s}$, where $Y$ is a Killing vector field on $S^{3}$. By a direct computation, 
we obtain the statement.

Now let us examine harmonic $i$-deformations of $\varphi_{s}$ which are not trivial.

3.1. The case of $s \neq 0, \pi / 6$

We decompose as $\operatorname{HID}\left(\varphi_{s}\right)=W_{0} \oplus W_{1}$, where $W_{1}=\boldsymbol{R} \xi_{7}$.

LEMma 3.3. Let $s \neq 0, \pi / 6$. Then a harmonic $i$-deformation of $\varphi_{\mathbf{s}}$ obtained by moving the parameter $s$ spans $W_{1}$.

Proof. We compute

$$
\begin{aligned}
\varphi_{s}^{-1} \frac{\partial \varphi_{s}}{\partial s} & =\left[\begin{array}{cc}
\cos s \cdot e^{-i t_{1}} & -\sin s \cdot e^{i t_{2}} \\
\sin s \cdot e^{-i t_{2}} & \cos s \cdot e^{i t_{1}}
\end{array}\right]\left[\begin{array}{cc}
-\sin s \cdot e^{i t_{1}} & \cos s \cdot e^{i t_{2}} \\
-\cos s \cdot e^{-i t_{2}} & -\sin s \cdot e^{-i t_{1}}
\end{array}\right] \\
& =\left[\begin{array}{cc}
0 & e^{-i t_{1}} e^{i t_{2}} \\
-e^{i t_{1}} e^{-i t_{2}} & 0
\end{array}\right]=\xi_{7} .
\end{aligned}
$$

Then we obtain the following.

Proposition 3.4. Let $s \neq 0, \pi / 6$. Any element of $\operatorname{HID}\left(\varphi_{s}\right)=W_{0} \oplus W_{1}$ is integrable.

Proof. We see that harmonic $i$-deformations of $\varphi_{s}$, which are induced by compositions of harmonic maps obtained by moving the parameter $s$ and isometries of $S U(2)$, span a direct sum $W_{0} \oplus W_{1}$.

3.2. The case of $s=\pi / 6$

We decompose as $\operatorname{HID}\left(\varphi_{\pi / 6}\right)=W_{0} \oplus W_{1} \oplus W_{2}$, where $W_{1}=\boldsymbol{R} \xi_{7}, W_{2}=\sum_{\imath=8}^{9} \boldsymbol{R} \boldsymbol{\xi}_{i}$. When $s=\pi / 6$, the statement of Lemma 3.3 also holds. Hence we obtain the following similar to Proposition 3.4.

PROposition 3.5. Any element of $W_{0} \oplus W_{1} \subset \operatorname{HID}\left(\varphi_{\pi / 6}\right)$ is integrable.

However, the integrability for an element of $\operatorname{HID}\left(\varphi_{\pi / 6}\right) \backslash\left(W_{0} \oplus W_{1}\right)$ can not be decided consequently. Then our next effort is to examine whether or not this element is integrable up to second order.

THEOREM 3.6. Any element of $\operatorname{HID}\left(\varphi_{\pi / 6}\right) \backslash\left(W_{0} \oplus W_{1}\right)$ is not integrable up to second order. Hence, it can not be integrable.

Proof. Let $\xi=\left[\begin{array}{cc}a & b \\ c & -a\end{array}\right] \in \operatorname{HID}\left(\varphi_{\pi / 6}\right) \backslash\left(W_{0} \oplus W_{1}\right)$ satisfying (2.7). Note that $b_{4}(=$ $\left.-\bar{c}_{4}\right) \neq 0$. By Lemma $1.4(1)$, we check whether or not 


$$
* P(\xi)=-\left[\left[\left(\theta_{\pi / 6}\right)_{1}, \xi\right], \frac{\partial \xi}{\partial t_{1}}\right]-\left[\left[\left(\theta_{\pi / 6}\right)_{2}, \xi\right], \frac{\partial \xi}{\partial t_{2}}\right]
$$

is orthogonal to $\operatorname{Ker} \mathscr{I}_{\varphi_{\pi / 6}}$. Choose

$$
\xi_{0}:=\left[\begin{array}{cc}
0 & -e^{-i t_{1}} e^{i t_{2}} \\
e^{i t_{1}} e^{-i t_{2}} & 0
\end{array}\right] \in \operatorname{Ker} \mathscr{T}_{\varphi_{\pi / 6}} .
$$

Then by long calculations, we obtain

$$
\int_{T_{2}}\left\langle * P(\xi), \xi_{0}\right\rangle_{\not \mathfrak{u}(2)}=-2\left(3-\tan ^{2} \frac{\pi}{6}\right) \tan \frac{\pi}{6} \times\left(2 b_{4} c_{4}\right)=\frac{32 \sqrt{3}}{9}\left|b_{4}\right|^{2} \neq 0 .
$$

Hence $\xi$ is not integrable up to second order. The second statement follows from Lemma 1.2 .

\subsection{The case of $s=0$}

We decompose as $\operatorname{HID}\left(\varphi_{0}\right)=V_{0} \oplus V_{1} \oplus V_{2}$, where $V_{1}=\sum_{\imath=6}^{?} \boldsymbol{R} \xi_{2}, V_{2}=\sum_{\imath=8}^{g} \boldsymbol{R} \tilde{\xi}_{\imath}$. Let us define two maps $\varphi_{s}^{\omega}, \tilde{\varphi}_{s}^{\omega}: T^{2} \rightarrow S U(2)(\boldsymbol{\omega} \in \boldsymbol{R})$ by

$$
\begin{aligned}
& \varphi_{s}^{\omega}\left(e^{i t_{1}}, e^{i t_{2}}\right):=\left[\begin{array}{cc}
\cos s \cdot e^{i t_{1}} & \sin s \cdot e^{\imath\left(t_{2}+\omega\right)} \\
-\sin s \cdot e^{-\imath\left(t_{2}+\omega\right)} & \cos s \cdot e^{-i t_{1}}
\end{array}\right], \\
& \tilde{\varphi}_{s}^{\omega}\left(e^{i t_{1}}, e^{i t_{2}}\right):=\left[\begin{array}{cc}
\cos s \cdot e^{i t_{1}} & \sin s \cdot e^{-\imath\left(t_{2}+\omega\right)} \\
-\sin s \cdot e^{\imath\left(t_{2}+\omega\right)} & \cos s \cdot e^{-\imath t_{1}}
\end{array}\right] .
\end{aligned}
$$

Then we see that $\varphi_{0}^{\omega}=\tilde{\varphi}_{0}^{\omega}=\varphi_{0}$ and that $\varphi_{s}^{\omega}, \tilde{\varphi}_{s}^{\omega}$ are harmonic maps in the same way to $(0.4)$.

LEMMA 3.7. Infinitesimal harmonic deformations of $\varphi_{s}^{\omega}$ (respectively, $\tilde{\varphi}_{s}^{\omega}$ ), which are obtained by fixing the parameter $\omega$ arbitrarily and moving the parameter $s$, span $V_{1}$ (respectively, $V_{2}$ ).

Proof. We see that

$$
\begin{aligned}
\left(\varphi_{s}^{\omega}\right)^{-1} \frac{\partial \varphi_{s}^{\omega}}{\partial s} & =\left[\begin{array}{cc}
\cos s \cdot e^{-i t_{1}} & -\sin s \cdot e^{\imath\left(t_{2}+\omega\right)} \\
\sin s \cdot e^{-\imath\left(t_{2}+\omega\right)} & \cos s \cdot e^{i t_{1}}
\end{array}\right]\left[\begin{array}{cc}
-\sin s \cdot e^{i t_{1}} & \cos s \cdot e^{i\left(t_{2}+\omega\right)} \\
-\cos s \cdot e^{-\imath\left(t_{2}+\omega\right)} & -\sin s \cdot e^{-i t_{1}}
\end{array}\right] \\
& =\left[\begin{array}{cc}
0 & e^{i \omega} e^{-i t_{1}} e^{i t_{2}} \\
-e^{-i \omega} e^{i t_{1}} e^{-i t_{2}} & 0
\end{array}\right] .
\end{aligned}
$$

By putting $\omega=0, \pi / 2$, it becomes $\xi_{7}, \xi_{6}$, respectively. Similarly, since

$$
\left(\tilde{\varphi}_{s}^{\omega}\right)^{-1} \frac{\partial \tilde{\varphi}_{s}^{\omega}}{\partial s}=\left[\begin{array}{lc}
\cos s \cdot e^{-i t_{1}} & -\sin s \cdot e^{-\imath\left(t_{2}+\omega\right)} \\
\sin s \cdot e^{\imath\left(t_{2}+\omega\right)} & \cos s \cdot e^{i t_{1}}
\end{array}\right]\left[\begin{array}{lc}
-\sin s \cdot e^{i t_{1}} & \cos s \cdot e^{-\imath\left(t_{2}+\omega\right)} \\
-\cos s \cdot e^{\imath\left(t_{2}+\omega\right)} & -\sin s \cdot e^{-i t} \cdot
\end{array}\right]
$$




$$
=\left[\begin{array}{cc}
0 & e^{-\imath \omega} e^{-i t_{1}} e^{-i t_{2}} \\
-e^{i \omega} e^{i t_{1}} e^{i t_{2}} & 0
\end{array}\right],
$$

putting $\omega=0,3 \pi / 2$, it becomes $\tilde{\xi}_{9}, \hat{\xi}_{8}$, respectively.

We discuss the integrability of harmonic $i$-deformations of $\varphi_{0}$. By Lemmas 3.2 and 3.7 , we have the following.

Proposition 3.8. Any element of $V_{0} \oplus V_{1}, V_{0} \oplus V_{2} \subset \operatorname{HID}\left(\varphi_{0}\right)$ is integrable.

Therefore our problem is to investigate whether or not an element of $\operatorname{HID}\left(\varphi_{0}\right) \backslash\left\{\left(V_{0} \oplus V_{1}\right) \cup\left(V_{0} \oplus V_{2}\right)\right\}$ is integrable.

THEOREM 3.9. Any element of $\operatorname{HID}\left(\varphi_{0}\right)$ is integrable up to second order.

Proof. Fix arbitrary $\xi=\left[\begin{array}{cc}a & b \\ c & -a\end{array}\right] \in \operatorname{HID}\left(\varphi_{0}\right)$ satisfying (2.8). Then we obtain that $\eta=\left[\begin{array}{cc}A & B \\ C & -A\end{array}\right] \in C^{\infty}(\underline{g})$ with $A \in \sqrt{-1} \boldsymbol{R}, B=-\bar{C}$ is a solution to $* \mathscr{T}_{\varphi_{0}}(\eta)+$ $P(\xi)=0$ if and only if each $A, B, C$ is of the form

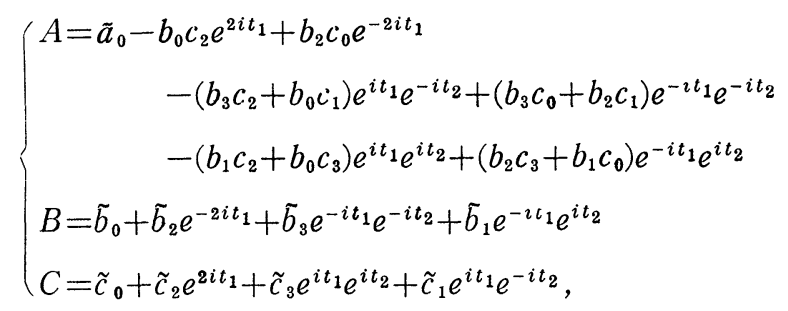

with $\tilde{a}_{0} \in \sqrt{-1} \boldsymbol{R}, \tilde{b}_{\imath}=-\overline{\tilde{c}}_{i} \in \boldsymbol{C}(i=0,1,2,3)$ chosen arbitrarily.

Unfortunately, Theorem 3.9 has not given any answer to our problem yet. Then let us proceed a more step.

Theorem 3.10. Any element of $\operatorname{HID}\left(\varphi_{0}\right) \backslash\left\{\left(V_{0} \oplus V_{1}\right) \cup\left(V_{0} \oplus V_{2}\right)\right\}$ is not integrable up to third order. Hence, it can not be integrable.

Proof. Let $\xi=\left[\begin{array}{cc}a & b \\ c & -a\end{array}\right] \in \operatorname{Ker} \mathscr{T}_{\varphi_{0}} \backslash\left\{\left(V_{0} \oplus V_{1}\right) \cup\left(V_{0} \oplus V_{2}\right)\right\}$ satisfying (2.8). Note that $b_{1}\left(=-\bar{c}_{1}\right) \neq 0$ and $b_{3}\left(=-\bar{c}_{3}\right) \neq 0$. Take also arbitrarily $\eta$ such that $(\xi, \eta)$ becomes a harmonic $i$-deformation of second order of $\varphi_{0}$, and then it satisfies (3.3). Our claim is to show $* Q(\xi, \eta)$ is not orthogonal to $\operatorname{Ker} \mathscr{I}_{\varphi_{0}}$. Choose

$$
\tilde{\xi}_{0}:=\left[\begin{array}{cc}
0 & b_{3} e^{-i t_{1}} e^{-i t_{2}}+b_{1} e^{-i t_{1}} e^{i t_{2}} \\
-\bar{b}_{3} e^{i t_{1}} e^{i t_{2}}-\bar{b}_{1} e^{i t_{1}} e^{-i t_{2}} & 0
\end{array}\right] \in \operatorname{Ker} \mathscr{I}_{\varphi_{0}} .
$$

Then by very long caluculations, we can show 


$$
\begin{aligned}
\int_{T_{2}}\left\langle * Q(\xi, \eta), \tilde{\xi}_{0}\right\rangle_{\mathfrak{z} \mathfrak{u}(2)} & =-12 b_{1} b_{3}\left(\bar{b}_{3} c_{1}+\bar{b}_{1} c_{3}\right)-12 c_{1} c_{3}\left(-b_{3} b_{1}-b_{1} b_{3}\right) \\
& =48\left|b_{1}\right|^{2}\left|b_{3}\right|^{2} \neq 0 .
\end{aligned}
$$

\section{$\S 4$. The structure of a neighborhood and the component in $\operatorname{Harm}\left(T^{2}, S^{3}\right)$}

Using the results in previous sections, we discuss the local structure of a neighborhood in $\operatorname{Harm}\left(T^{2}, S^{3}\right)$ around $\varphi_{s}$.

Proposition 4.1. Let $0 \leqq s \leqq \pi / 4$. For $s \neq 0$, a neighborhood in $\operatorname{Harm}\left(T^{2}, S^{3}\right)$ around $\varphi_{s}$ becomes a 7-dimensional smooth manifold. On the other hand, the space $\operatorname{Harm}\left(T^{2}, S^{3}\right)$ has a singular point at $\varphi_{0}$.

Remark. Although nonintegrable harmonic $i$-deformations occur at $s=\pi / 6$, a neighborhood in $\operatorname{Harm}\left(T^{2}, S^{3}\right)$ around $\varphi_{\pi / 6}$ becomes a smooth manifold.

Let $0 \leqq s \leqq 2 \pi$. We need to give attention to the case of $s=\pi / 2$. It follows from Lemma 3.7 that the families $\varphi_{s}^{\omega}$ and $\tilde{\varphi}_{s}^{\omega}$ intersect orthogonally each other at $\varphi_{0}$. Similarly, we observe that up to deformations obtained by moving the parameter $\omega, \varphi_{s}^{\omega}$ and $\hat{\varphi}_{s}^{\omega}$ intersect orthogonally each other at $\varphi_{\pi / 2}$. Here $\hat{\varphi}_{s}^{\omega}$ : $T^{2} \rightarrow S U(2)(\boldsymbol{\omega} \in \boldsymbol{R})$ is a family of harmonic maps defined by

$$
\hat{\varphi}_{s}^{\omega}\left(e^{i t_{1}}, e^{i t_{2}}\right):=\left[\begin{array}{cc}
\cos s \cdot e^{-\imath t_{1}} & \sin s \cdot e^{\imath\left(t_{2}+\omega\right)} \\
-\sin s \cdot e^{-\imath\left(t_{2}+\omega\right)} & \cos s \cdot e^{i t_{1}}
\end{array}\right] .
$$

Finally let us determine the connected component in $\operatorname{Harm}\left(T^{2}, S^{3}\right)$ containing $\varphi_{s}$ and discuss its compactness. We define a new family $\bar{\varphi}_{s}^{\omega}: T^{2} \rightarrow S U(2)$ $(\boldsymbol{\omega} \in \boldsymbol{R})$ of harmonic maps defined by

$$
\bar{\varphi}_{s}^{\omega}\left(e^{i t_{1}}, e^{i t_{2}}\right):=\left[\begin{array}{cc}
\cos s \cdot e^{-i t_{1}} & \sin s \cdot e^{-\imath\left(t_{2}+\omega\right)} \\
-\sin s \cdot e^{\imath\left(t_{2}+\omega\right)} & \cos s \cdot e^{i t_{1}}
\end{array}\right] .
$$

THEOREM 4.2. The set of harmonic maps from $T^{2}$ into $S^{3}$ obtained by acting the group $S O(4)$ on the union of four families $\left\{\varphi_{s}^{\omega}\right\} \cup\left\{\tilde{\varphi}_{s}^{\omega}\right\} \cup\left\{\hat{\varphi}_{s}^{\omega}\right\} \cup\left\{\bar{\varphi}_{s}^{\omega}\right\}$ with $0 \leqq$ $s \leqq 2 \pi$ becomes a connected component in $\operatorname{Harm}\left(T^{2}, S^{3}\right)$ containing $\varphi_{s}$. Hence, the connected component is compact.

Figure 4.1 indicates how four family $\left\{\varphi_{s}^{\omega}\right\},\left\{\tilde{\varphi}_{s}^{\omega}\right\},\left\{\hat{\varphi}_{s}^{\omega}\right\}$ and $\left\{\bar{\varphi}_{s}^{\omega}\right\}$ with $0 \leqq s \leqq$ $2 \pi$ of harmonic maps intersect orthogonally each other, up to deformations with respect to the parameter $\omega$.

Remark. The action of $S O(4)$ moves $\left\{\varphi_{s}^{\omega}\right\}$ to $\left\{\bar{\varphi}_{s}^{\omega}\right\}$ and $\left\{\tilde{\varphi}_{s}^{\omega}\right\}$ to $\left\{\hat{\varphi}_{s}^{\omega}\right\}$. Then the union of two circles $\left[\varphi_{s}^{\omega}\right]$ and $\left[\tilde{\varphi}_{s}^{\omega}\right]$, which intersect orthogonally each other at $\left[\varphi_{0}\right]$, becomes the connected component in the quotient space $\operatorname{Harm}\left(T^{2}, S^{3}\right) /$ $S O(4)$ containing $\left[\varphi_{s}\right]$. 


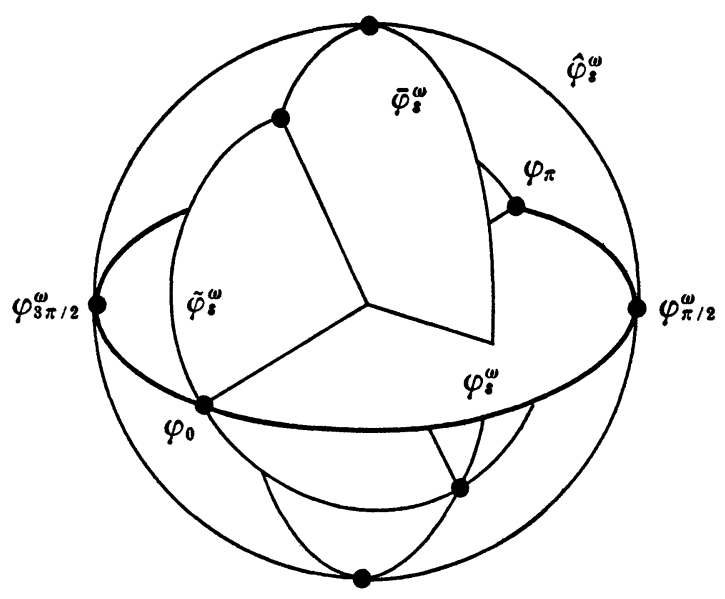

Figure 4.1.

\section{REFERENCES}

[EL] J. Eells AND L. Lemaire, Deformations of metrics and associated harmonic maps, Geometry and Analysis, Papers Dedicated to Memory of V.K. Patodi, Springer, 1981, 33-45.

[Ej] N. EJIRI, The homotopically energy minimizing harmonic maps of tori into $R P^{3}(1)$, preprint.

[H] N.J. Hitchin, Harmonic maps from a 2-torus to the 3-sphere. J. Differential Geom., 31 (1990), 627-710.

[K1] N. KoIso, Variation of harmonic mapping caused by a deformation of Riemannian metric, Hokkaido Math. J., 8 (1979), 199-213.

[K2] N. Kolso, Rigidity and infinitesimal deformability of Einstein metrics, Osaka J. Math., 19 (1982), 643-668.

[K3] N. KoIso, Einstein metrics and complex structures, Invent. Math., 73 (1983), 71-106.

[S] T. SunADA, Rigidity of certain harmonic mappings, Invent. Math., 51 (1979), 297-307.

[T] G. Tотн, On rigidity of harmonic mappings into spheres, J. London Math. Soc., 26 (1982), 475-486.

Department of Mathematics

Tokyo Metropolitan University

Minami-Ohsawa 1-1, Hachioji

TOKYO 192-03, JAPAN

E-mail:mmukai@math.metro-u.ac.jp 